\title{
Utility of TIVA in COVID-19 Era
}

\author{
Poonam Malhotra Kapoor ${ }^{1}$ Tushar Chokshi \\ ${ }^{1}$ Department of Cardiac Anaesthesia, CTC, AlIMS, New Delhi, India \\ 2Department of Anaesthesia, Starling Hospital, Vadodara, \\ Gujarat, India
}

J Card Crit Care:2021;5:1-4

\section{COVID-19 Thrombosis Is Unprecedented}

An unsolved mystery, the coronavirus disease 2019 (COVID19) pandemic, which has taken the world by surprise, is clearly, as evidenced last few months, procoagulation and thrombotic in etiology. It is a collision of coagulation, inflammatory, and complementary pathways. There are multiple reports and publications that this COVID-19 pandemic is associated with both arterial and venous thrombosis and pulmonary embolism, with marked increase in clots in extracorporeal circuits as well. ${ }^{1}$

\section{The Intensive Care Unit in the "COVID-19 Era"}

The COVID-19 pandemic has been an unsolved mystery. The COVID-19 cytokine storm has affected more men than women. ${ }^{2,3}$ Nothing lasts forever and these testing times will pass eventually. We need to put our resources to good use and focus on delivering a sustainable level of care. Once the disease process is well understood and logistics catered to, the disease tide will ebb as it has. As an anesthesiologist and critical care intensivist, the use of right drug therapy becomes an important day-to-day challenge in the COVID-19 times.

\section{Anesthetic Considerations for Rationalizing Drug Use in the Operating Theater in COVID- 19 Era Exist}

Hospitals are facing an unprecedented surge in intensive care unit (ICU) patients because 5 to $10 \%$ of COVID-19 patients deteriorate rapidly and require prolonged intensive care for acute respiratory distress syndrome (ARDS). ${ }^{4}$ These patients tend to require high doses of sedatives and paralytics. This has resulted in increasing worldwide demand for critical care
Address for correspondence Poonam Malhotra Kapoor, MD, DNB, MNAMS, FIACTA, FTEE, FISCU, Department of Cardiac Anaesthesia, CTC, AIIMS, New Delhi, 110029, India (e-mail: drpoonamaiims@gmail.com).

drugs, including and not limited to sedatives, paralytics, opioids, and vasoactive agents, especially to support ventilatory strategies for ARDS such as proning and paralysis. ${ }^{5}$

The high number of patients infected with the severe acute aspiratory syndrome coronavirus 2 (SARS-CoV-2) virus requiring care for ARDS puts sedation in the critical care unit to the egde. ${ }^{4}$

Rationalizing drug dosage in the operation theater and ICU becomes essential in the wake of these more than 3 million COVID-19 patients. The latter suffer from adult respiratory distress syndrome and require prolonged ventilatory days in the ICU. So, the requirement for sedatives hypnotics will markedly increase. This is where the role of total intravenous anesthetic (TIVA) agents becomes essential to the anesthesiologist to combat COVID-19 pandemic.

\section{TIVA in COVID-19 Era}

In perspective of COVID-19, TIVA is far more advantageous than inhalational anesthesia. It avoids the deleterious effects of immunosuppression and lacks any respiratory irritation, thus providing an edge in the current situation. Many peripheral surgeries can be done with the patient breathing spontaneously without any airway device, thus avoiding airway instrumentation leading to droplet and aerosol generation. IV agents can be utilized to provide sedation during regional anesthesia, which can easily be escalated to contain pain due to sparing of blocks or receding neuraxial anesthesia.

\section{Types of TIVA-How Is TIVA Initiated?}

TIVA may be provided either with insertion of endotracheal tube or supra glottic airway device, nasal, or oral airway or oxygen alone without insertion of any airway device (-Fig. 1).
DOI https://doi.org/ $10.1055 / \mathrm{s}-0041-1728229$ ISSN 2457-0206 (c)2021. Official Publication of The Simulation Society (TSS), accredited by International Society of Cardiovascular Ultrasound (ISCU).

This is an open access article published by Thieme under the terms of the Creative Commons Attribution-NonDerivative-NonCommercial-License, permitting copying and reproduction so long as the original work is given appropriate credit. Contents may not be used for commercial purposes, or adapted, remixed, transformed or built upon. (https://creativecommons.org/licenses/by-nc-nd/4.0/).

Thieme Medical and Scientific Publishers Pvt. Ltd. A-12, 2nd Floor, Sector 2, Noida-201301 UP, India 


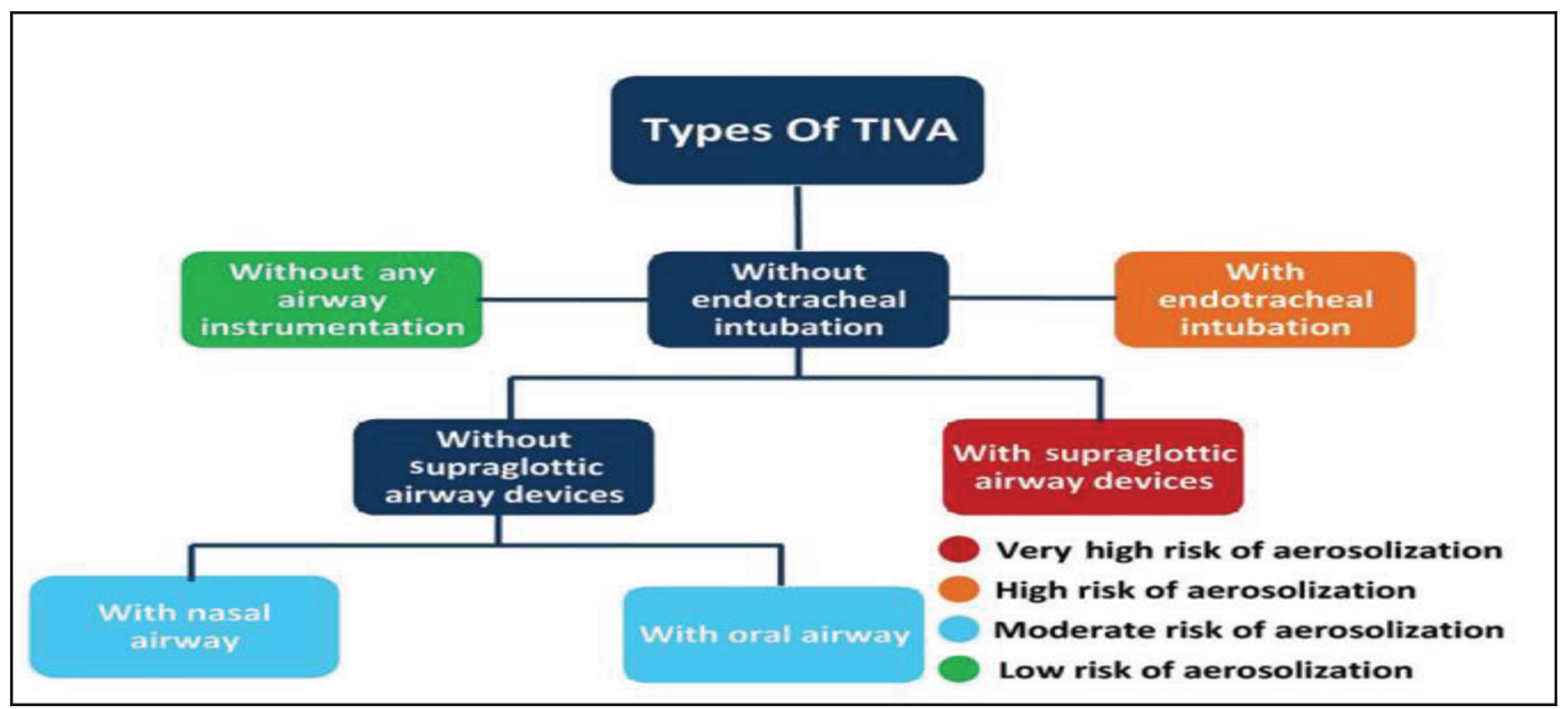

Fig. 1 Types of total intravenous anesthesia (TIVA).

\section{Advantages of TIVA}

Multiple advantages of TIVA exist, some of which are highlighted in - Table 1 but limitation of TIVA too should be kept in mind.

\section{Selective TIVA Drugs Useful in Pandemic: Use of Ketamine and Rocuronium}

Low-dose ketamine, rocuronium, and vecuronium as IV anesthesia agents are helpful in viral infection to offset both

Table 1 Advantages of TIVA over inhalational agents in COVID-19

\begin{tabular}{|l|l|}
\hline$>$ Patient is unaware \\
\hline$>$ Minimal aerosol generation \\
\hline$>$ No mask over face \\
\hline$>$ Minimal effect on immune system \\
\hline$>$ No sudden concentration of gas or vapor \\
\hline$>$ No risk of malignant hyperthermia \\
\hline$>$ Less postoperative delirium and emergence phenomena \\
\hline$>$ Better operating conditions \\
\hline$>$ Less operating room pollution \\
\hline$>$ Reduced stress response \\
\hline$>$ Better preservation of cerebral auto regulation \\
\hline$>$ Decreased postoperative nausea and vomiting \\
\hline$>$ Avoidance of cross infection \\
\hline$>$ Minimal intervention to airway in many surgeries \\
\hline$>$ Wide acceptance across age groups \\
\hline$>$ Definite advantages in remote locations \\
\hline
\end{tabular}

Abbreviations: COVID-19, coronavirus disease 2019; TIVA, total intravenous anesthesia. the inflammatory lesion and aid in ultra-fast tracking in many cardiac surgeries like off-pump coronary artery bypass grafting. Although vecuronium is a gold standard in cardiac surgery as a neuromuscular blocking agent, rocuronium bromide can be advocated as the drug of choice in elective as well as emergency cardiac surgery where rapid intubation will be beneficial without compromise of hemodynamic stability. ${ }^{5,6}$

\section{Merits of TIVA during COVID-19 Pandemic (-Fig. 2)}

A. Hypertriglyceridemia: A potential side effect of propofol sedation in critical illness should be kept in mind.

B. Extubation of patient with COVID-19 should be cautions.

Extubation is an aerosol-generating procedure, commonly associated with coughing, which necessitates proximity of the anesthetist to the patient. Extubation should ideally take place in a negative pressure room, if available. All nonessential staff should exit the room before extubation. Personal protective equipment with airborne precautions is required during extubation and for personnel entering the room for a variable period of time after extubation, dependent on room ventilation (-Fig. 3).

Limit the need for subsequent staff interactions with prophylactic antiemetics; adequate analgesia; consider regional anesthesia; perform oropharyngeal suction with vigilance, as this may generate aerosols. Antitussive drugs, such as remifentanil, lidocaine, and dexmedetomidine, reduce the risk of coughing and minimize agitation on extubation.

\section{Challenges in COVID-19 Met with the Use of Alternative Agents and Considerations}

If general anesthesia cannot be avoided, anesthesiologists are encouraged to use other drugs not commonly used in ICU, for 


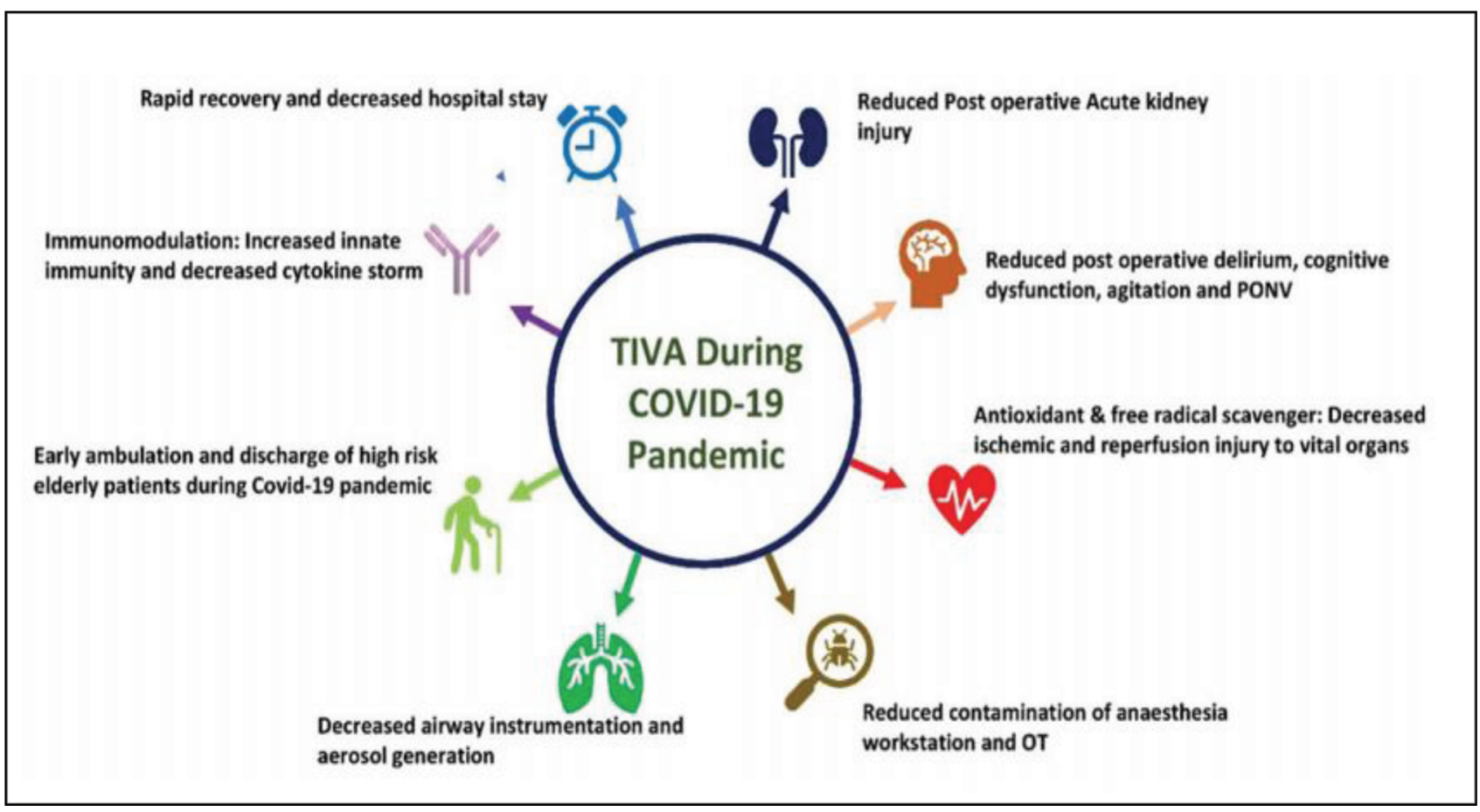

Fig. 2 Merits of total intravenous anesthesia (TIVA) during coronavirus disease 2019 (COVID-19) pandemic. OT, operation theater.

Table 2 Limitations of TIVA

$>$ The major limitation with TIVA includes pain on injection, especially true with propofol, though that limitation is reduced with administration of agents such as lidocaine/opioids and using wide bore cannula

$>$ The other challenging situation may arise in cases of difficult cannulation, though this will be equally challenging in cases of providing anesthetic agents via maintenance with inhalational agents

$>$ Other limitations include the risk of bacterial contamination, especially when there are frequent misconnections in line

Abbreviation: TIVA, total intravenous anesthesia.

example, ketamine, nitrous oxide, volatiles, thiopentone, and etomidate. Oral premedications like lorazepam or gabapentin were used to reduce anesthetic requirements. Multimodal analgesia was optimized with nonopioid adjuncts, such as IV lignocaine infusion, magnesium, dexamethasone, clonidine, ketamine, and nonsteroidal anti-inflammatory drugs.

\section{Regional Anesthetic Techniques and Opioid-Sparing Strategies Should Be Adopted More Robustly in the COVID-19 Patient}

Regional techniques can provide adequate anesthesia in majority of limb and lower abdominal surgery and reduce sedative and opioid use and oxygen utilization compared with general anesthesia.

\section{Ethical Considerations in TIVA}

Ethical considerations in TIVA is another challenge in Covid era. Modifications are needed in anaesthesia techniques especially during induction and extubation. It is a known fact that inadequate regional anaesthesia does more harm than good. Drug rationalization is a more appropriate term than drug conservation. Adhering to all safety issues during intravenous infusion techniques is essential. Training in TIVA should be a part of core anaesthesia training post covid era, to ensure competency and safety in TIVA use (-Fig. 3)

\section{Conclusion}

Patient safety and clinical management require the use of sedation in most SARS-CoV-2 patients with IV induction, which is essential induction of anesthesia.

Use of neuromuscular blockers for intubation and extubation techniques avoiding aerosol generation and nasal prongs becomes mandatory in this covid pandemic. Myths such as using Extracorporeal membrane oxygenation (ECMO) or PEEP in covid era should be busted with sound scientific knowledge? Do we manage patients with a wet or dry circuit for mucus plugging and secretion management? Time and experience will be narrative! ${ }^{7}$ Sedation analgesia for diagnostic/therapeutic procedures should be adopted. Adequate sedation should be extended in cardiac practice also in the cath laboratory for monitored anaesthesia care, percutaneous procedures for device insertion and all hybrid procedures in operating room and MRI suites and also in intensive medication for sedation and analgesia, outside the operating room. The judicious use of TIVA in all fields of cardiac care practice is here to stay post covid era. 


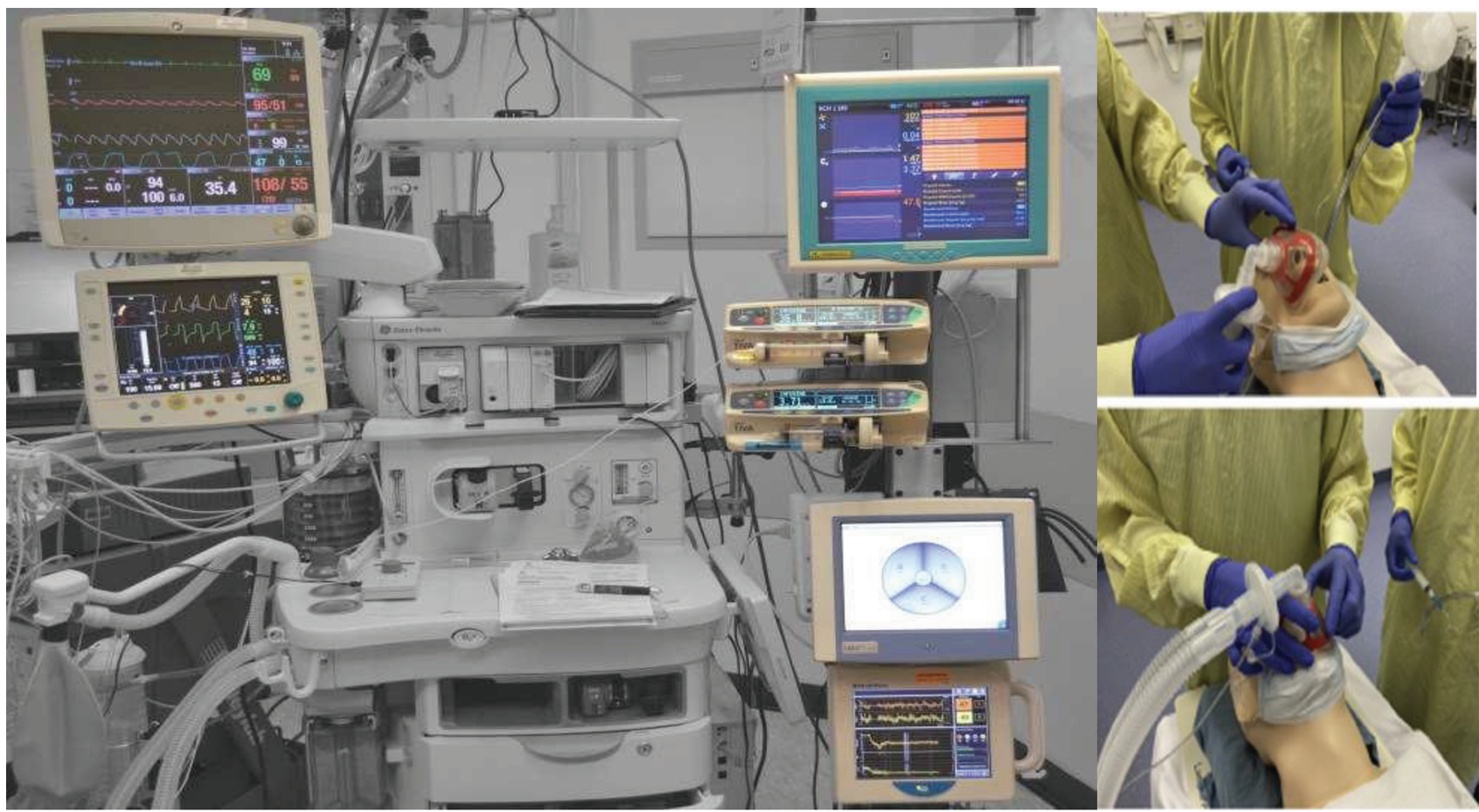

Fig. 3 An array of TIVA infusion pumps with the anaesthesia machine and monitors using intubations and for safe extubation too!

\section{Conflict of Interest}

None declared.

\section{References}

1 Poonam MKE. COVID-19 thrombosis: an unsolved mystery. J Card Crit Care 2020;4:1-4

2 Malhotra Kapoor P. Editorial: women in COVID-19 pandemic: influential leadership is the need of the hour. Indian J Cardiovasc Dis Women WINCARS 2020;5(3):271-273

3 Chokshi T, Channabasappa S, Vergheese DC, Bajwa SJ, Gupta $\mathrm{B}$, Mehdiratta L. Re-emergence of TIVA in COVID times. Indian J Anaesth 2020;64(14, Suppl 2) :S125-S131

4 Longrois D, Petitjeans F, Simonet O, et al. Clinical practice: should we radically alter our sedation of critical care patients, especially given the COVID-19 pandemics? Rom J Anaesth Intensive Care 2020;27(2):43-76

5 Poston JT, Patel BK, Davis AM. Management of critically ill adults with COVID-19. JAMA 2020;323(18):1839-1841

6 Mezher M, Taylor NP. FDA reports shortage of sedation drug used for putting COVID-19 patients on ventilators [internet]. Available from URL: https://www.raps.org/news-and-articles/ news-articles/2020/4/fda-reports-shortage-of-sedation-drugused-for-put. Accessed March 22, 2021

7 Sharma A, Sharan S, Kapoor PM, Chowdhury UK. COVID19 ECMO myths busted. Journal of Cardiac Critical Care TSS. 2020;4(1):51-55

8 Malhotra Kapoor P. Editorial: is resilience in COVID-19, its lasting legacy? Journal of Cardiac Critical Care TSS 2020; $4(2): 73-074$ 